\title{
Genomic organization and promoter activity of the maize starch branching enzyme I gene
}

\author{
Kyung-Nam Kim ${ }^{1}$, Dane K. Fisher ${ }^{2}$, Ming Gao ${ }^{3}$, Mark J. Guiltinan ${ }^{1,2,3}$ * \\ Intercollege Graduate Programs in Plant Physiology and Genetics, The Biotechnology Institute, and Department of Horticulture, \\ The Pennsylvania State University, University Park, Pennsylvania, PA 16802, USA
}

Received 17 February 1998; received in revised form 26 May 1998; accepted 1 June 1998; Received by A. Dugaiczyk

\begin{abstract}
Starch branching enzymes (SBE) which catalyse the formation of $\alpha$-1,6-glucan linkages are of crucial importance for the quantity and quality of starch synthesized in plants. In maize (Zea mays L.), three SBE isoforms (SBEI, IIa and IIb) have been identified and shown to exhibit differential expression patterns. As a first step toward understanding the regulatory mechanisms controlling their expression, we isolated and sequenced a maize genomic DNA $(-2190$ to +5929$)$ which contains the entire coding region of SBEI (Sbel) as well as 5'-and 3'-flanking sequences. Using this clone, we established a complete genomic organization of the maize Sbel gene. The transcribed region consists of 14 exons and 13 introns, distributed over $5.7 \mathrm{~kb}$. A consensus TATA-box and a G-box containing a perfect palindromic sequence, CCACGTGG, were found in the 5'-flanking region. Genomic Southern blot analysis indicated that two Sbel genes with divergent $5^{\prime}$-flanking sequences exist in the maize genome, suggesting the possibility that they are differentially regulated. A chimeric construct containing the 5 -flanking region of Sbel $(-2190$ to +27$)$ fused to the $\beta$-glucuronidase gene (pKG101) showed promoter activity after it was introduced into maize endosperm suspension cells by particle bombardment. (C) 1998 Elsevier Science B.V. All rights reserved.
\end{abstract}

Keywords: Zea mays L.; Starch biosynthesis; G-box; Particle bombardment

\section{Introduction}

Starch, the major storage carbohydrate of most higher plants, is synthesized in both photosynthetic and nonphotosynthetic cells and can be divided into two types, transitory and reserve, which are differentially utilized by plants. A typical example of transitory starch can be found in photosynthetic organs such as leaves, in which starch is accumulated in chloroplasts during the day and is mobilized for translocation at night. Transitory starch can also be synthesized in the non-photosynthetic

\footnotetext{
* Corresponding author. Tel.: (814) 863 7957; Fax: (814) 863 1357; E-mail: mjg9@psu.edu

${ }^{1}$ Present address: Department of Plant and Microbial Biology, 451 Koshland, University of California, Berkeley, CA 94720, USA.

${ }^{2}$ Present address: Monsanto Corporation, 700 Chesterfield Parkway North, Chesterfield, MO 63198, USA.

${ }^{3}$ Present address: Plant Biotechnology Institute, NRC, 110 Gymnasium Place, Saskatoon, SK, S7N 0W9, Canada.
}

Abbreviations: DAP, days after pollination; PCR, polymerase chain reaction; pfu, plaque-forming units; SBE, starch branching enzyme; Sbe, gene encoding SBE. tissues such as meristems, pollen grains and root cap cells. The major site of reserve starch accumulation is in the amyloplasts of storage organs including seeds, fruits and tubers (reviewed in Shannon and Garwood, 1984; Martin and Smith, 1995). Starch also provides carbon and energy for vegetative and reproductive development and plays a very important role in buffering the level of soluble sugar via a diurnal starch-sucrose interconversion cycle (Stitt et al., 1987). According to the starch-statolith theory, starch is also involved in sensing gravity (reviewed in Salisbury, 1993).

Starch is not only a major source of food and feed but also is an important industrial commodity throughout the world. It is utilized in the production of ethanol, paper, adhesives, textiles and biodegradable plastics, not to mention its uses in the food industry. Other uses include building and packaging materials as well as a pharmaceutical delivery system (reviewed in White, 1994). Depending on the industrial applications of starch, however, its desired properties vary. For example, starch with high amylose content is required for adhesives and films, while high amylopectin starch is used for salad dressings. 
Starch is found as a water-insoluble granule consisting of crystalline and amorphous regions and is mainly composed of two different polysaccharides, amylose and amylopectin (Takeda et al., 1988). Amylose is considered to be an essentially linear $\alpha$-1,4-linked glucose chain of about 1000 residues long and is associated only with the amorphous regions. In contrast, amylopectin is a more highly branched macromolecule consisting of linear $\alpha$-1,4-glucose chains with $\alpha$-1,6-glucosidic bonds at branch points. Branches occur about every 21 glucose residues (Kainuma, 1988). Amylopectin is present in both areas but is considered to be solely responsible for the crystallinity of the starch granule. Usually, amylose and amylopectin constitute about $23-31 \%$ and $69-77 \%$ of starch, respectively. However, the proportion can vary considerably depending on starch source (Shannon and Garwood, 1984). Since the two polysaccharides, amylose and amylopectin, have distinctive properties, it is apparent that the ratio of amylose and amylopectin is important in determining many of the chemical and physical properties of starch.

Starch branching enzymes (EC 2.4.1.28) have been shown to catalyse the formation of amylopectin by introducing $\alpha-1,6$ branch points into the linear $\alpha-1,4$-linked glucose chains. Recent genetic and biochemical evidence has shown that starch debranching enzyme (EC 2.4.1.41), which hydrolyzes $\alpha-1,6$ glycosidic bonds, also plays an important role in producing the final branching pattern of amylopectin (James et al., 1995). Apparently, the balanced actions of starch branching enzymes (SBE) and debranching enzymes are critical in determining the final degree of branching in amylopectin. Although ADP-glucose pyrophosphorylase (EC 2.7.7.23) is known to catalyse a rate-limiting reaction in starch biosynthesis, SBEs also facilitate starch synthesis by increasing the number of non-reducing ends, the sites of glucose addition by starch synthases (EC 2.4.1.21). Thus, SBEs are of crucial importance for the quantity and quality of starch synthesized in the plant (Edwards et al., 1988). In fact, mutation in Sbe genes of pea, maize and rice severely decreased total starch content and changed the ratio of amylose and amylopectin (Shannon and Garwood, 1984; Smith, 1988; Mizuno et al., 1993).

Multiple forms of SBEs have been identified and characterized biochemically from various plants, including rice, spinach, pea, potato, teosinte, and maize (reviewed by Preiss, 1991; Martin and Smith, 1995). In maize, three SBE isoforms (SBEI, IIa and IIb) were separated by anion-exchange chromatography (Boyer and Preiss, 1978a,b). SBEI is quite different from SBEIIa and IIb in molecular size, biochemical and enzyme kinetic properties, and immunological reactivity. In contrast, SBEIIa and IIb are very similar, but not identical, in many biochemical and immunological properties (reviewed in Preiss, 1991; Fisher et al., 1996). Guan and
Preiss (1993) showed that SBEI prefers amylose to amylopectin as substrate in vitro, whereas SBEIIa and $\mathrm{IIb}$ preferentially catalyse amylopectin. This led to the hypothesis that SBEI produces slightly branched molecules, which would then serve as substrates for the action of SBEIIa and IIb. In this way, each SBE isoform may be responsible for a unique aspect of amylopectin biosynthesis and structure. Despite the well-characterized biochemical properties of each SBE isoform in maize, how each SBE isoform contributes to the synthesis of the amylopectin fraction of starch in vivo is still poorly understood.

Isolation of the maize cDNAs encoding SBE isoforms (Sbe1, $2 a$ and $2 b$ ) enabled the investigation of the Sbe genes at molecular level (Fisher et al., 1993, 1995; Gao et al., 1996, 1997). Fisher et al. (1996) determined that SBEIIa and IIb are the products of separate genes, and Gao et al. $(199,1997))$ demonstrated that the Sbe genes are differentially expressed during kernel development and in various tissues, suggesting that they play distinct roles in starch biosynthesis. As a first step toward understanding the regulatory mechanisms controlling Sbe gene expression, we isolated a full-length maize genomic DNA fragment containing the entire Sbel gene. Structural and functional analysis of this gene reported in this manuscript revealed a complete genomic organization and demonstrated the transcriptional activity of its promoter region.

\section{Materials and methods}

\subsection{PCR amplification}

Maize (Zea mays L., inbred W64A) genomic DNA prepared from 22-DAP kernels using the method of Rogers and Bendich (1985) was amplified in a 50-ml reaction mixture containing $1 \mathrm{mg}$ of target DNA, $1 \mathrm{mM}$ of each primer, $200 \mathrm{mM}$ of each dNTP, $10 \mathrm{mM}$ Tris- $\mathrm{HCl}(\mathrm{pH} 8.3), 50 \mathrm{mM} \mathrm{KCl}, 1.5 \mathrm{mM} \mathrm{MgCl} 2,0.01 \%$ gelatin $(\mathrm{w} / \mathrm{v})$, and $2.5 \mathrm{U}$ of Taq DNA polymerase (Boehringer Mannheim, Indianapolis, IN, USA). The mixture without dNTP was overlaid with $50 \mathrm{ml}$ of mineral oil (Sigma, St. Louis, MO, USA) and incubated for $5 \mathrm{~min}$ at $94^{\circ} \mathrm{C}$. The dNTPs were added to the mixture at the end of the incubation and then the mixture was cycled 35 times in a thermal cycler (ERICOMP, San Diego, CA, USA) as follows: $94^{\circ} \mathrm{C}$ for $30 \mathrm{~s} ; 55^{\circ} \mathrm{C}$ for $1 \mathrm{~min}$; and $72^{\circ} \mathrm{C}$ for $2 \mathrm{~min}$; with a final $72^{\circ} \mathrm{C}$ extension of $7 \mathrm{~min}$. The primers were designed according to the published sequence data of the maize partial Sbel cDNA (Baba et al., 1991). The 5' primer, 5'-GACTGAATTCCTGCGCAGGAGGCAGAGCTT-3', and the 3' primer, 5'-GATCGAATTCCATAGATACGTGGAGCAGCA-3', are homologous and complementary to DNA sequences of the maize Sbel cDNA 
from 438 to $457 \mathrm{bp}$ and from 745 to $764 \mathrm{bp}$, respectively. Each primer contains an EcoRI restriction enzyme site and four extra nucleotides (underlined) at their $5^{\prime}$ ends for convenience of subsequent cloning of the PCR product. After amplification, $15 \mathrm{ml}$ of the reaction sample was run on an agarose gel $(1.5 \%, \mathrm{w} / \mathrm{v})$ in $1 \times \mathrm{TAE}$ buffer containing $0.04 \mathrm{M}$ Tris-acetate and $1 \mathrm{mM}$ EDTA. A single PCR product of approx. $0.5 \mathrm{~kb}$ was detected on an ethidium bromide-stained agarose gel, digested with EcoRI restriction enzyme and cloned into the corresponding site of pBluescript $\mathrm{SK}^{-}$(Stratagene, $\mathrm{La}$ Jolla, CA, USA) creating plasmid pB1.

\subsection{Maize genomic library screening and DNA sequencing}

An EMBL-3 genomic library (Clonetech, Palo Alto, CA, USA) prepared from maize seedlings (two-leaf stage, B73) was screened essentially according to Sambrook et al. (1989). Approximately $3 \times 10^{5} \mathrm{pfu}$ were transferred onto nylon membranes (Hybond-N+, Amersham, UK) and hybridized with the ${ }^{32} \mathrm{P}$-labeled Sbel genomic PCR product excised from $\mathrm{pB} 1$. Hybridization was performed at $55^{\circ} \mathrm{C}$ for $20 \mathrm{~h}$ in $0.5 \mathrm{M}$ $\mathrm{Na}_{2} \mathrm{HPO}_{4}$ ( $\mathrm{pH} 7.2$ ) and 7\% SDS (Church and Gilbert, 1984 ) with gentle agitation at 40 cycles per minute on a rotary shaker. Following the hybridization, filters were washed twice in 5\% SEN (5\% SDS (w/v), 1 mM EDTA, $\left.0.04 \mathrm{M} \mathrm{Na}_{2} \mathrm{HPO}_{4}, \mathrm{pH} 7.2\right)$ and once in $1 \%$ SEN (1\% SDS, $1 \mathrm{mM}$ EDTA, $0.04 \mathrm{M} \mathrm{Na}_{2} \mathrm{HPO}_{4}, \mathrm{pH} 7.2$ ) for $15 \mathrm{~min}$ at $65^{\circ} \mathrm{C}$. Plaques strongly hybridizing to the probe were selected and purified through three rounds of screening. Phage DNAs were isolated from the positive plaques according to Chisholm's method (Chisholm, 1988) and were digested with PstI to release the inserts from the EMBL-3 vector. The restriction fragments were separated on a $0.8 \%$ agarose gel and blotted onto nylon membranes. The blots were probed with ${ }^{32}$ P-labeled full-length Sbel cDNA (Fisher et al., 1995) as described above and hybridizing DNA fragments were identified and subcloned into pBluescript $\mathrm{SK}^{-}$. DNA sequences were determined by the dideoxynucleotide chain termination method (Sanger et al., 1977) with Sequenase Version 2.0 (United States Biochemical, Cleveland, OH, USA). Sequence analyses were performed using programs from DNASTAR (Madison, WI, USA).

\subsection{Primer extension analysis}

To locate the transcription initiation site of the Sbel gene, an oligonucleotide, 5'-GGCGACACGAGGCACAGCAT-3', which is complementary to the sense strand sequence of the Sbel cDNA from +1 to +20 relative to the translation start site (ATG) was radiolabeled at its $5^{\prime}$ terminus with $\mathrm{T} 4$ polynucleotide kinase and $\gamma_{-}{ }^{32}$ P-ATP (Sambrook et al., 1989). Approximately $10^{5} \mathrm{cpm}$ of the labeled primer was hybridized at $35^{\circ} \mathrm{C}$ with $10 \mu \mathrm{g}$ of total RNA, which was isolated from 30-DAP maize kernels (B73) according to the protocol of Vries et al. (1988). After hybridization for $8 \mathrm{~h}$, complementary DNA was synthesized from the annealed primer by the addition of reverse transcriptase and dNTP. Following the addition of EDTA and RNAase A into the reaction, the nucleic acid was precipitated with ethanol. The reaction products were resuspended in sequencing gel loading buffer, denatured at $95^{\circ} \mathrm{C}$, electrophoresed through a $5 \%$ polyacrylamide sequencing gel $(\mathrm{w} / \mathrm{v})$, and visualized by autoradiography. In order to provide size markers, part of the Sbel gene was sequenced with the same primer used in the primer extension experiment.

\subsection{3'-rapid amplification of cDNA ends (3'-RACE)}

To isolate the 3'-end of the Sbel transcript, the 3'RACE method was used (Frohman et al., 1988). Firststrand cDNA synthesis reaction was performed as follows: $14 \mathrm{ml}$ of a mixture containing $5 \mu \mathrm{g}$ of total RNA from 30-DAP maize kernels (B73) and 50 pmol of a 39bp oligonucleotide with $17 \mathrm{dT}$ residues and an adaptor sequence, 5'-GGTCGACTCGAGTCGACATCGATTTTTTTTTTTTTTTTT-3', was heated at $70^{\circ} \mathrm{C}$ for $10 \mathrm{~min}$ and quickly chilled on ice. To the chilled sample, $2 \mu \mathrm{l}$ of $10 \times$ synthesis buffer $(200 \mathrm{mM}$ Tris $-\mathrm{HCl}(\mathrm{pH}$ 8.4), $500 \mathrm{mM} \mathrm{KCl}, 25 \mathrm{mM} \mathrm{MgCl}$, and $1 \mathrm{mg} / \mathrm{ml} \mathrm{BSA}$ ), $1 \mathrm{ml}$ of $10 \mathrm{mM}$ dNTP mix, $2 \mathrm{ml}$ of $0.1 \mathrm{M}$ DTT and $1 \mathrm{ml}$ (200 U) of SUPERSCRIPT reverse transcriptase (GIBCO BRL, Grand Island, NY, USA) were added and incubated at room temperature for $10 \mathrm{~min}$. The reaction mixture was then placed in a $42^{\circ} \mathrm{C}$ water bath for $50 \mathrm{~min}$ and transferred to a $90^{\circ} \mathrm{C}$ water bath. After $5 \mathrm{~min}$ incubation, $1 \mathrm{ml}$ of RNase $\mathrm{H}(2 \mathrm{U} / \mathrm{ml})$ was added and incubation at $37^{\circ} \mathrm{C}$ was continued for $20 \mathrm{~min}$. Next, the first-strand cDNA obtained was amplified directly by the PCR method using a gene-specific primer (5'GACTGAGCTCATACCAAATGAAGCCAGGAG-3'), which is homologous to sequence of the Sbel gene from +5382 to +5401, and an adaptor primer (5'GGTCGACTCGAGTCGACATCGA-3'). After amplification, a single DNA band detected on an agarose gel $(1.5 \%, \mathrm{w} / \mathrm{v})$ was isolated and digested with $\mathrm{SacI}$ and $X h o I$ (underlined within the primers). The resulting fragment, approx. $350 \mathrm{bp}$ in length, was cloned into a pBlusScript $\mathrm{SK}^{-}(\mathrm{pB} 13 \mathrm{R})$ and sequenced.

\subsection{Genomic DNA blot analysis}

Maize genomic DNA was prepared from 7-day-old etiolated seedlings (inbred B73) according to the method described by Junghans and Metzlaff (1990). Ten micrograms of genomic DNA was digested with restriction 
enzymes BamHI, EcoRI, BglI, and HindIII, separated on $0.8 \%$ agarose gels, and transferred onto nylon membranes (Hybond-N, Amersham, UK) in $20 \times \mathrm{SSC}$ containing $3 \mathrm{M} \mathrm{NaCl}$ and $0.3 \mathrm{M}$ sodium citrate $(\mathrm{pH} 7.0)$ according to Sambrook et al. (1989). The DNA was crosslinked to the membrane by 3.5 min of UV irradiation on a transilluminator $(312 \mathrm{~nm})$. The genomic blots were prehybridized at $65^{\circ} \mathrm{C}$ for $1 \mathrm{~h}$ in $0.5 \mathrm{M} \mathrm{Na}_{2} \mathrm{HPO}_{4}$ ( $\mathrm{pH} \mathrm{7.2),} \mathrm{7 \%} \mathrm{SDS,} \mathrm{and} 100 \mu \mathrm{g} / \mathrm{ml}$ denatured salmon sperm DNA. Using the random primed DNA labeling kit (Boehringer Mannheim, Indianapolis, IN, USA), $25 \mathrm{ng}$ of a full-length Sbel cDNA (Fisher et al., 1995) was labeled with $\left[\alpha-{ }^{32} \mathrm{P}\right]$-dCTP. This labeled probe was added to the prehybridization solution and incubated at $65^{\circ} \mathrm{C}$ for $18 \mathrm{~h}$. Blots were washed twice in 5\% SEN and once in $1 \% \mathrm{SEN}$ for $15 \mathrm{~min}$ at $65^{\circ} \mathrm{C}$ and were exposed to Kodak X-AR film at $-80^{\circ} \mathrm{C}$ for $48 \mathrm{~h}$ using two intensifying screens.

\subsection{Construction of $\mathrm{Sbe} 1$ promoter-UidA expression plasmids}

To make a transcriptional chimeric construct consisting of the Sbel promoter $(-2190$ to +27$)$ fused to a $\beta$-glucuronidase (GUS) reporter gene, UidA, a BamHI restriction enzyme site was created just before the translation initiation site of the Sbel gene as follows: the DNA sequence between -253 and +27 of the Sbe1 gene was amplified via polymerase chain reaction using $P f u$ DNA polymerase (Stratagene, La Jolla, CA, USA) to enhance the fidelity of PCR amplification. The 5' primer, 5'-CCAGCTCCACGGTTGTT CGTGT-3', is homologous to sequence of the Shel gene from -253 to -232 . An ApaI restriction enzyme site (GGGCCC) is located immediately downstream of the 5'-primerbinding region of the Sbel promoter, -203 to -198 . The 3' primer, 5'-CGATGGATCC TGTGACGGCGTGTGAGT CCC-3', consists of a DNA sequence complementary to that of the Sbel gene from +8 to +27 and a BamHI restriction enzyme site (GGATCC) flanked with four random nucleotides (underlined). The PCR product was digested with ApaI and BamHI, and the resulting 236-bp fragment was cloned into pBluescript $\mathrm{SK}^{-}$and sequenced in order to verify that no misincorporation had occurred in the DNA sequence during the PCR amplification. To incorporate this mutation into the context of a longer promoter fragment, the Sbel genomic clone (25-1-1) was digested with $S a l I$ to isolate the approximately $3.0-\mathrm{kb}$ fragment, which was then blunt-ended with Klenow and ligated with Pst I linkers (New England Biolabs, Beverley, MA, USA). After complete digestion with $P s t \mathrm{I}$ and $A p a \mathrm{I}$, the resulting 2-kb Sbel promoter fragment was ligated to the 236-bp ApaI-BamHI fragment and cloned into plasmid pBI221 cut with PstI and BamHI, thereby creating plasmid pKG101.

\subsection{Transient gene expression assay}

Suspension culture cells of maize endosperm (inbred A636), kindly provided by J.L. Anthony (DEKALB Genetics Corporation, Mystic, CT) were grown in 250-ml large-mouth Erlenmeyer flasks containing $80 \mathrm{ml}$ of Murashige and Skoog basal salt medium (Murashige and Skoog, 1962) supplemented with $0.4 \mathrm{mg} / 1$ thiamine, $2 \mathrm{~g} / 1$ asparagine, and $30 \mathrm{~g} / 1$ sucrose (Shannon and Liu, 1977). The culture was maintained in the dark at $29^{\circ} \mathrm{C}$ on a rotary shaker $(120 \mathrm{rpm})$ and was subcultured every 7 days by transferring a portion of the cell suspension into fresh medium. For particle bombardment, the growing cells ( 3 days after subculture) were evenly distributed over the surface of three layers of filter paper (Whatman No. $4,55 \mathrm{~mm}$ in diameter) moistened with $3 \mathrm{ml}$ of the liquid medium and positioned in the middle of a $10-\mathrm{cm}$ Petri dish. Three milligrams of $1.6-\mu \mathrm{m}$ gold particles were coated with $10 \mu \mathrm{g}$ of pKG101 according to the method described by Xu et al. (1996) and introduced into the cells using a Bio-Rad PDS-1000/He Biolistic Particle Delivery system. Bombardments were performed at $650 \mathrm{psi}$ under a vacuum of 26 inches of $\mathrm{Hg}$ with a distance of $10 \mathrm{~cm}$ between the cells and the microprojectile launch site of the particle gun. Following the bombardments, the Petri dishes were sealed with parafilm and then incubated in the dark at $25^{\circ} \mathrm{C}$ for $24 \mathrm{~h}$.

\subsection{Histochemical GUS staining}

Histochemical assays of GUS activity were performed according to the method described by Jefferson (1987) with minor modifications. Briefly, after $24 \mathrm{~h}$ incubation, cells on the filter paper were transferred into Petri dishes containing $2 \mathrm{ml}$ of GUS staining solution $(50 \mathrm{mM}$ sodium phosphate buffer ( $\mathrm{pH} 8.0$ ), $0.05 \mathrm{mM}$ potassium ferricyanide, $0.05 \mathrm{mM}$ potassium ferrocyanide, $0.2 \%$ Triton X-100 (w/v), 20\% methanol (v/v), and $1 \mathrm{mg} / \mathrm{ml}$ 5-bromo-4-chloro-3-indolyl- $\beta$-D-glucuronide). The Petri dishes were then sealed and incubated at $37^{\circ} \mathrm{C}$ overnight. The cells were examined and photographed under a Nikon SMZ-U dissecting microscope.

\section{Results}

\subsection{PCR amplification of maize genomic DNA}

In order to obtain a DNA probe for genomic library screening, maize genomic DNA prepared from 22-DAP kernels (W64A) was amplified by polymerase chain reaction (PCR) using upper and lower primers designed to anneal the Sbel cDNA (Baba et al., 1991) from 438 to 457 and 745 to 764 , respectively. A single amplified DNA band, approximately $450 \mathrm{bp}$ in length, was observed on an ethidium bromide-stained agarose gel. 
The PCR product containing EcoRI sites at both 5'and 3 '-ends was digested with EcoRI and the resulting fragment was cloned and sequenced.

Alignment of sequences between the maize PCR genomic fragment and the published Sbel cDNA (Baba et al., 1991) shows that the PCR fragment contained the predicted Sbel cDNA sequence with only three nucleotide differences and a 103-bp intron (data not shown). The differences could be explained by cultivar polymorphisms or misincorporation of bases during PCR amplification by Taq DNA polymerase which does not have proofreading activity. This PCR fragment was ${ }^{32} \mathrm{P}$-labeled and used as a hybridization probe for screening a maize genomic library to isolate Sbel genomic clones.

\subsection{Isolation and analysis of a maize Sbe1 genomic clone}

In a screen of approximately $3 \times 10^{5}$ plaque-forming units from a genomic library prepared from maize seedlings (inbred B73), eight positive lambda clones were isolated, which strongly hybridized to the probe. Restriction mapping and partial DNA sequencing of these clones indicated they all probably originated from the same genetic locus. A full-length clone (25-1-1), containing the entire coding region of the Sbel gene, as well as $5^{\prime}$ - and $3^{\prime}$-flanking sequences, was selected for further analyses. Fig. 1A shows a restriction map of the genomic clone. The $3.0-\mathrm{kb}$ SalI fragment and the $6.2-\mathrm{kb}$ PstI fragment from the clone were subcloned into pBluescript $\mathrm{SK}^{-}$producing plasmids $\mathrm{pBI} 5-1$ and $\mathrm{pBI} 5-$ 2 , and their nucleotide sequences were completely determined.

A

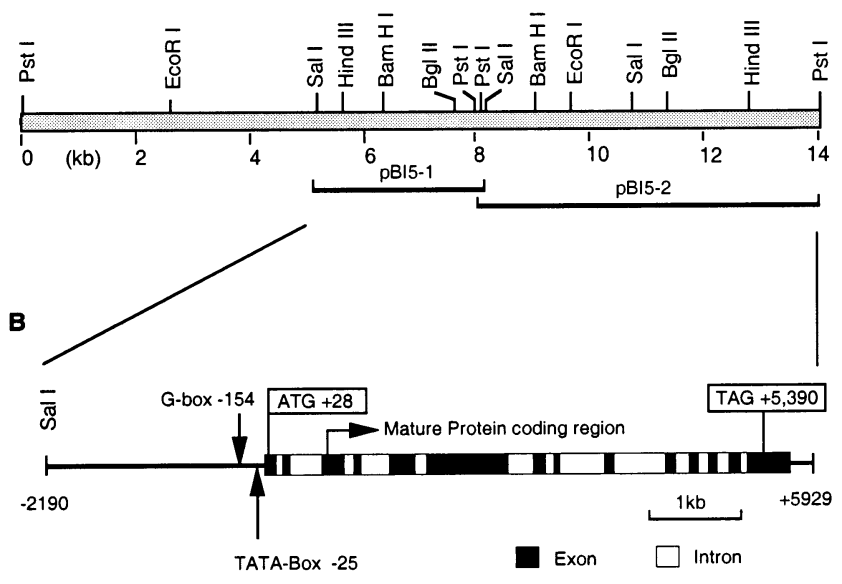

Fig. 1. Structure of the lambda clone 5-1-1 containing the Sbel gene. (A) Restriction map of the 5-1-1 clone. pBI5-1 and pBI5-2 indicate subclones in plasmid pBluescript $\mathrm{SK}^{-}$. (B) Genomic structure of the Sbe1 gene. The thin black lines indicate the $5^{\prime}$ - or 3 '-flanking sequences of the Sbe1 gene. The solid black boxes indicate exons and the open boxes denote introns. The numbers represent positions relative to the transcription initiation site $(+1)$.
A consensus TATA-box, as well as a G-box containing a perfect palindromic sequence known as a G-box, CCACGTGG, were found in the 5'-flanking region of the gene (Fig. 2). Primer extension analysis displayed a single extended product of 44 nucleotides (Fig. 3), which co-migrates with an A residue in the sequencing ladder generated with the Sbel 5'-flanking region. This indicates that consistent with many eukaryotic genes, the transcription initiates at a position which is located $25 \mathrm{bp}$ downstream from the putative TATA box, suggesting that the TATA box may be a functional element of the Sbel promoter.

To determine the polyadenylation site of the Sbe1 gene, 3'-RACE was conducted. It demonstrated that a poly (A) tail occurs at an adenine nucleotide located $29 \mathrm{bp}$ downstream from a putative polyadenylation signal (AATAAA) in the Sbel gene (data not shown). Along with the primer extension result, this indicated that the transcribed region in the Sbel gene is $5690 \mathrm{bp}$ in length.

\subsection{Structure of the Sbe1 genomic clone}

Alignment of the genomic sequence with the published maize Sbel cDNA sequence (Fisher et al., 1995) revealed that the gene is composed of 14 exons and 13 introns distributed over $5.7 \mathrm{~kb}$. Fig. 1B summarizes the organization of the maize Sbel gene. The cDNA sequence is identical to the corresponding genomic sequence except for a 1-bp mismatch in exon 14. Table 1 shows the sequences around the exon/intron junctions and a list of putative branch point consensus sequences, which were derived as described by Brown (1986). The introns, relatively AT-rich (61\%) compared with the exons (52\%), vary in length from 73 to $565 \mathrm{bp}$, all of which have the conserved sequences at their $5{ }^{\prime}$ - and $3^{\prime}$-ends, following the 'GT - AG' rule of plant introns (Brown, 1986). Exon 1, containing 27 bp of 5'-untranslated DNA sequence, and exon 2 occur in the transit peptide region which may be essential for transporting the gene product into the amyloplast. Exon 14 contains the translation stop codon (TGA) and 3'-untranslated region, as well as the putative polyadenylation signal (AATAAA). The exons vary in length from 63 to $907 \mathrm{bp}$.

Comparison of the maize and rice Sbel genomic DNA sequences (Kawasaki et al., 1993b) revealed two large highly conserved regions in the $5^{\prime}$-flanking sequences (Fig. 2 and Fig. 4A). One region, 161 bp in length, was present between -2190 and -1890 in the maize Sbel 5 -flanking sequence, and has $82 \%$ similarity with the corresponding rice region. The other, $342 \mathrm{bp}$ in length, is located from -1804 to -1611 , and shows $85 \%$ similarity with the corresponding rice region. These sequences conserved between the species suggest that the regions may play an important role in gene expres- 
gtcgact gecetctagaccccgat gaagtcaaggatagtgagcgeccaaccaagt t taggctcccccagccagatgatcatgt taggtgt tccactgccataaaagaaagcgtccgtgat gtcgtact tgggggeggtgaatacgtaaggggacagatgctcatataccttcatctgttt tactcgaaat tactaatgttt tccctatg tcagctaaagt gaaatctcacccgaccaatc t tgcagat tgtgaat gact tgaagt t taccgtgaaccatgct gt ggaacccat caat gaaaagct gcacat gat at cc gagaacat caaaaagcgt gagaagggaaagaggaaaac gaat gat gatagc tgcatcagt tcaccaacatcct tgaccagggtcatcagtgggat tgat gat cegctcctgaacggaagct tgagtgacaacagcggtccaaagaaagcacggaggcagcge aggaaatcaggc tat gcaagcgcagagagcggt ggggagagcagcgaccatggcc taggcgggtt tgagatccgagggaacagat ggat tactagggagt gacagct aggagcaat ct gt - 1591

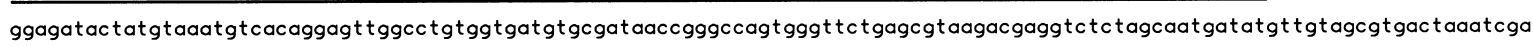

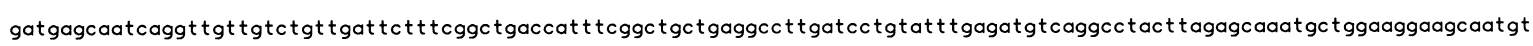

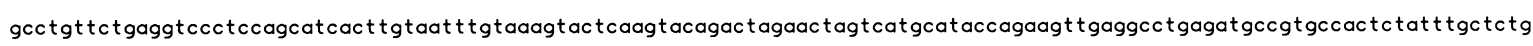
tt tcatgct tgccgt tat tatatcactatt ttcactgttcat tctaaggcegttgtcacacgggtaccagaagagcagaacacctttt tacgttccttt gctgctatggatggact tc gagagttccttgagaaagcactctcatctatct tccaat tccttgtt tccttgtggatcctgatcactgaagatggccatt gatttttcacttttcagt tatatataaagtgaagacg - 991

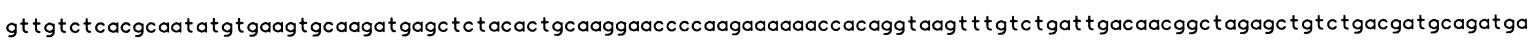

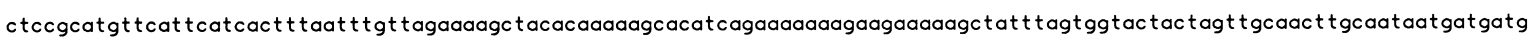
ataaatct gcacaagccatagctatgctatatgctatagctatg tatatg tacacaaaaaa tacattttttgtgctattttttt taccgctagtataatatccatgtct tgc tacaacac

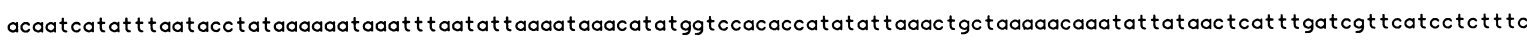
ggt tagtgaggtggacagtgagagcgct gcatcgtgt tat gggtt tgactggtt tctcacggctcatctgtgt tg taacgacctatctatggtcaaacaaact at taggat tat tgt ta -39 1 ggc gaaaaat gagggaga taaa taaacc ta taagcaggacacat gaaacat a t get t taaacag tagagat taacaaca taaaa aaaaaaaggcaaacc gggaagcg t gcaagcccaaa ggccaatcggcccagaaccagctccacggt tgt tcgtgtccgcccacgtggcacgcccggccat tcc gggeccacagat ccggctcagggtcatgtgccact gccat ggccccetcct tg tcgcagcggcagat tgcgacggggaagaaaggt gaggagaccaagc gaaaaaaat cacgct t tcat tgcgaggggggaggatagggaggaagacgccaagccage tccagt cc ggcaccc gatatadagcggcaggcact tggat tgctgACGAGATGGGACTCACACGCCGTCACAATGCTGTGCCTCGTGTCGCCCTCTTC TATA-box
TCGGATGCTGATCGGGCGGCACCGCCGGGGATCGCG. . INTRON 1 ...GGTGGCGGCAATGTGCGCCTGAGTGTGTTGTCTGTCCAGTGCAAGGCTCGCCGGTCAGGGGTGCGGAAG.
$S$ G . INTRON 2...GTCAAGAGCAAATTCGCCACTGCAGCTACTGTGCAAGAAGATAAAACTATGGCAACTGCCAAAGGCGATGTCGACCATCTCCCCATATACGACCTGGACCCCAAGCT

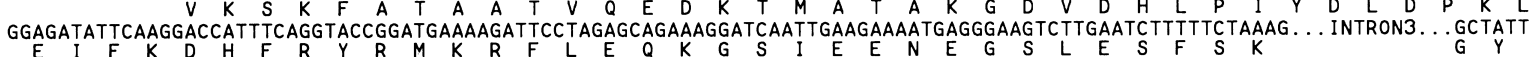
TGAAATTTGGGATTAATACAAATGAGGATGGAACTGTATATCGTGATGGGCACCTGCTGCGCA. I INTRON 4 . . GGAGGCAGAGCTTATTGGTGACTTCAATGACTGGAATGGTGC + 1413 AAACCATAAGATGGAGAAGGATAAATTTGGTGTTTGGTCGATCAAAATTGACCATGTCAAAGGGAAACCTGCCATCCCTCACAATTCCAAGGTTAAATTTCGCTTTCTACATGGTGGAGT

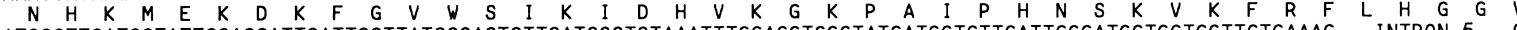
ATGGGTTGATCGTATTCCAGCATTGATTCGTTATGCGACTGTTGATGCCTCTAAATTTGGAGCTCCCTATGATGGTGTTCATTGGGATCCTCCTGCTTCTGAAAG. . INTRON 5. . . G TACACATTTAAGCATCCTCGGCCTTCAAAGCCTGCTGCTCCACGTATCTATGAAGCCCATGTAGGTATGAGTGGTGAAAAGCCAGCAGTAAGCACATATAGGGATTTGCAGACAATGTG TTGCCACGCATACGAGCAAATAACTACAACACAGTTCAGTTGATGGCAGTTATGGAGCATTCGTACTATGCTTCTTTCGGGTACCATGTGACAAATTTCTTTGCGGTTAGCAGCAGATCA + 1982 PA A GGCACACCAGAGGACCTCAAATATCTTGTTGATAAGGCACACAGTTTGGGTTTGCGAGTTCTGATGGATGTTGTCCATAGCCATGCAAGTAATAATGTCACAGATGGTTTAAATGGCTAT

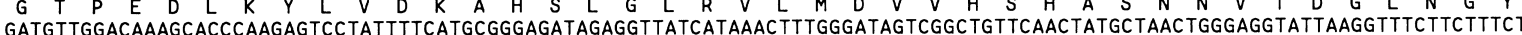

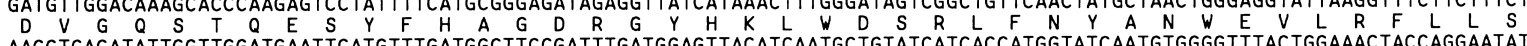
AACCTGAGATATTGGTTGGATGAATTCATGTTTGATGGCTTCCGATTTGATGGAGTTACATCAATGCTGTATCATCACCATGGTATCAATGTGGGGTTTACTGGAACTACTCAGGAATAT TTCAGTTTGGACACAGCTGTGGATGCAGTTGTTTACATGATGCTTGCAAACCATTTAATGCACAAACTCTTGCCAGAAGCAACTGTTGTTGCTGAAGATGTTTCAGGCATGCCGGTCCTT + 2462

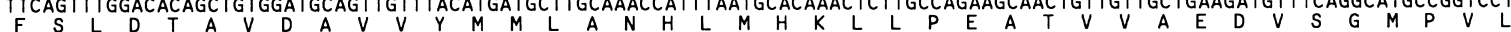
TGCCGGCCAGTTGATGAAGGTGGGTTGGGTTTGACTATCGCCTGGCAATGGCTATCCCTGATAGATGGATTGACTACCTGAAGAATAAAGATGACTCTGAGTGGTCGATGGGTGAAATA GCGCATACTTTGACTAACAGGAGATATACTGAAAAATGCATCGCATATGCTGAGAGCCATGATCAG. . INTRON 6. . TCTATTGTTGGCGACAAAACTATTGCATTTCTCCTGATGG

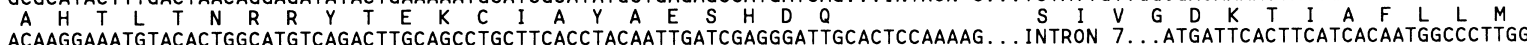
D K E M Y T G M S D L Q P A S P T I D R G I A L Q K . AGGTGATGGCTACTTGAATTTTATGGGAAATGAG... INTRON 8...TTTGGTCACCCAGAATGGATTGACTTTCCAAGAGAAGGGACAACTGGAGCTATGATAAATGCAGACGACAG +3747 G D G Y L N F M G N E F TGGAGCCTTGTGGACACTGATCACTTGCGGTACAAG... INTRON 9...TACATGAATGCGTTTGACCAAGCGATGAATGCGCTCGATGAGAGATTTTCCTTCCTTTCGTCGTCAAAGC W S L V V D T D H L R Y K N AGATCGTCAGCGACATGAACGATGAGGAAAAG... INTRON $10 \ldots$. GTTATTGTCTTTGAACGTGGAGATTTAGTTTTTGTTTTCAATTTCCATCCCAAGAAAACTTACGAGGG. . IN

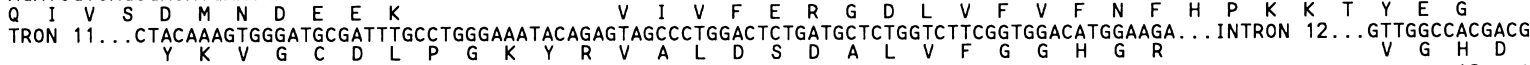
TGGATCACTTCACGTCGCCTGAAGGGGTGCAGGGGTGCCCGAAACGAACTTCAACAACCGGCCGAACTCGTTCAAAGTCCTTTCTCCGCCCCGCACCTGTGTG... INTRON 13... G +5198 $V$ D H F T S P E G V P G V P E T N F N N R P N S F K V L S P P R T C V CTTATTACCGTGTAGACGAAGCAGGGGCTGGACGACGTCTTCACGCGAAAGCAGAGACAGGAAAGACGTCTCCAGCAGAGAGCATCGACGTCAAAGCTTCCAGAGCTAGTAGCAAAGAAG $A Y Y Y R$ V D E A G A G R R L H A K A E T G K T S P A E S I D V K A S R A S S K E ACAAGGAGGCAACGGCTGGTGGCAAGAAGGGATGGAAGTTTGCGCGGCAGCCATCCGATCAAGATACCAAATGAAGCCAGGAGTCCTTGGTGAGGACTGGACTGGCTGCCGGCGCCCTG D K E A T A G G K K G W K F A R Q P S D Q D T K * TAGAATAATAATCAGGGATGGATGGATGGTGTGTATTGGCTATCTGGCTAGACGTGCATGTGCCCAGTTTGTATGTACAGGAGCAGTTCCCGTCCAGAATAAAAAAAAACTTGTTGGGG +5678

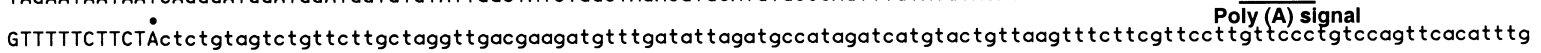
at tccagctgttcagcaggceggtcagctcagctccacaccgggggccaggccggcgtcacgcaggatcttttcatttcatgcgtcataacacaaacacttt gatcttt tc +5910

Fig. 2. Nucleotide sequence of the Sbel gene and 5'- and 3'-flanking regions. The GenBank accession number is AF072724. Flanking regions are shown in lower-case letters, while exons are presented in upper-case letters. The deduced amino acid sequences are shown below the string of exon sequences. Numbers indicate the distance relative to the transcription start site $(+1)$ which is indicated by the arrowhead. The consensus TATA and G-box sequences, as well as the putative polyadenylation signal are underlined. The region containing at least $82 \%$ sequence homology with the rice Sbe1 5'-flanking region is also underlined. The asterisk and dot indicate the stop codon and putative polyadenylation site, respectively.

sion. Interestingly, a part of the latter region from -1804 to -1611 (194 bp in length) shares $83 \%$ similarity with the 5'-untranslated sequence of a
$\mathrm{Ca}^{2+}$-dependent protein kinase gene in rice (Kawasaki et al., 1993a), suggesting that a maize version of the protein kinase gene may be located immediately 


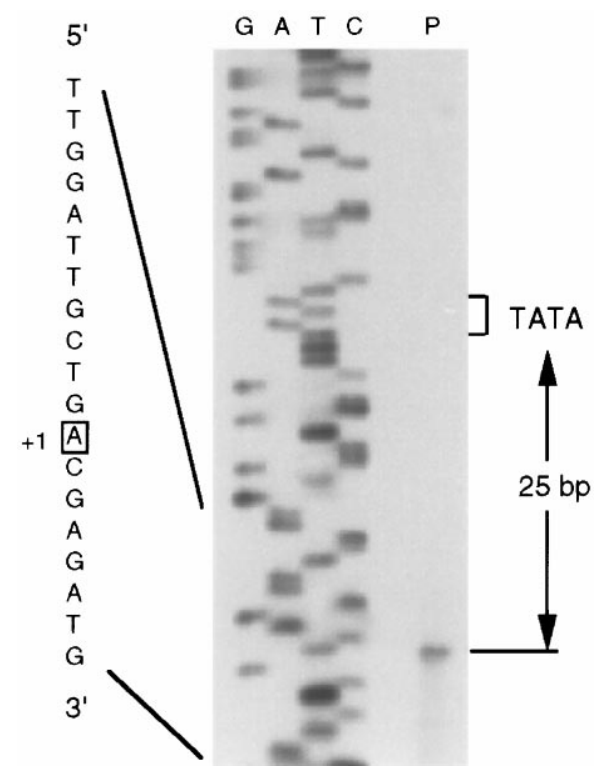

Fig. 3. Primer extension analysis of the transcription initiation site of the Sbel gene. A ${ }^{32} \mathrm{P}$-end-labeled antisense primer was annealed to $10 \mu \mathrm{g}$ of total RNA isolated from maize kernels (30 DAP) and extended using reverse transcriptase. The extended cDNA product was then analyzed on a sequencing gel (lane P) along with a Sbel 5 '-flanking genomic DNA sequencing ladder generated by the same primer. The number inside the vertical double-headed arrow on the right refers to distance from TATA-box to the primer extension product indicated by the horizontal arrow. The sense sequence around the product is shown on the left and the transcription initiation site is boxed and assigned +1 .

upstream of the Sbel gene. In the rice genome, the two genes are separated by approximately $1.4 \mathrm{~kb}$, and transcribed divergently from each other (Fig. 4A).

5 -flanking sequences downstream from the two conserved regions did not show sequence similarity between the two genes (less than 25\%) except for a G-box motif
A

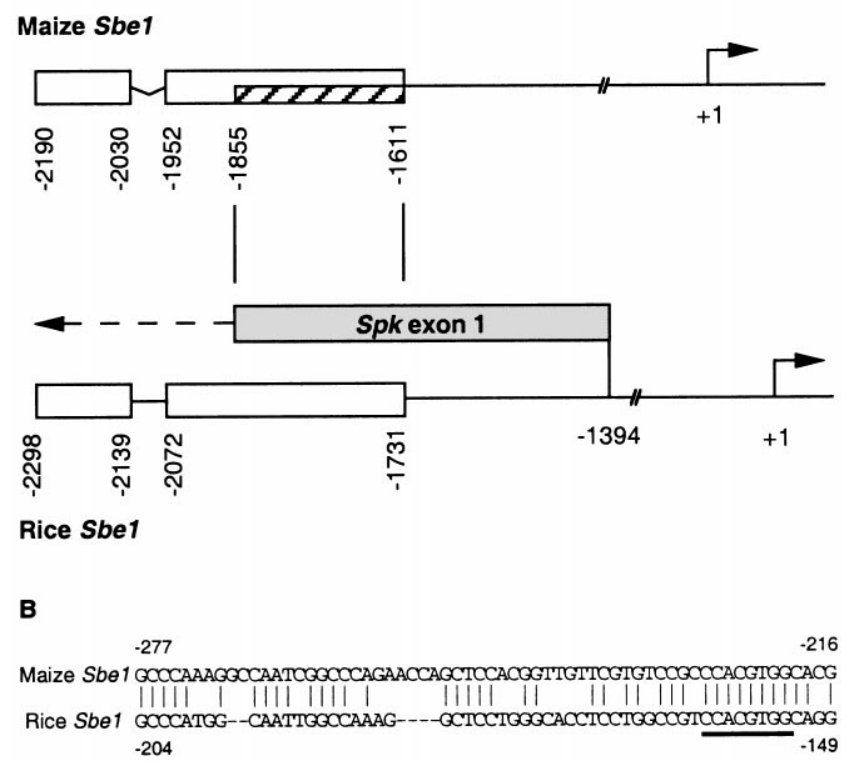

Fig. 4. Sequence comparison of the maize and rice Sbe1 promoters. (A) Highly conserved sequences found in the distal promoter regions. The open boxes indicate the conserved regions containing at least $82 \%$ sequence similarity between the two Sbel promoters. The striped box denotes $83 \%$ similarity between the maize Sbel and the rice Spk $\left(\mathrm{Ca}^{\not z}\right.$-dependent protein kinase) cDNA sequence which is represented by the stippled box. The arrows indicate transcription directions and the numbers indicate the distance relative to the relevant transcription start sites. (B) Proximal sequences showing conservation between the two Sbe1 promoters. Short vertical lines indicate conserved nucleotides and dashes represent gaps to maximize aligment. The G-box is underlined.

and several small segments adjacent to the G-box (Fig. 4B). This may indicate that the G-box plays a role in regulation of Sbel gene expression. The G-box is found in other plant genes which respond to diverse

Table 1

List of introns and sequences of exon/intron borders in the Sbel gene

\begin{tabular}{|c|c|c|c|c|c|}
\hline $\begin{array}{l}\text { Intron } \\
\text { number }\end{array}$ & Exon/ & Putative intron branch point ${ }^{\mathrm{a}}$ & /Exon & $\begin{array}{l}\text { Intron } \\
\text { size (bp) }\end{array}$ & $\begin{array}{l}\text { GC content } \\
(\%)\end{array}$ \\
\hline 1 & TCGCG & GTAAG .... CTGAT..1 $17^{\mathrm{b}} \cdot \mathrm{CGCAG}$ & GGTGG & 82 & 46.3 \\
\hline 2 & GGAAG & $\overline{\overline{\mathrm{GT}} \mathrm{AGA}} \ldots . . \mathrm{C} \overline{\mathrm{T}} \mathrm{G} \overline{\mathrm{A}} \mathrm{A} . .24 . . \mathrm{GGA} \overline{\mathrm{AG}}$ & GTCAA & 377 & 36.9 \\
\hline 3 & TAAAG & GTTAG .... ATCAT..30..TTCAG & GCTAT & 120 & 34.2 \\
\hline 4 & GCGCA & 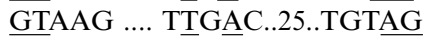 & GGAGG & 319 & 34.2 \\
\hline 5 & GAAAG & 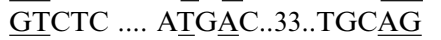 & GTACA & 103 & 35.9 \\
\hline 6 & ATCAG & 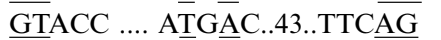 & TCTAT & 262 & 32.1 \\
\hline 7 & AAAAG & 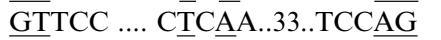 & ATGAT & 97 & 39.2 \\
\hline 8 & ATGAG & 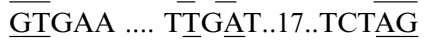 & TTTGG & 488 & 35.2 \\
\hline 9 & ACAAG & GTTAT .... CTAAC..37..AAC $\overline{A G}$ & TACAT & 565 & 37.3 \\
\hline 10 & AAAAG & 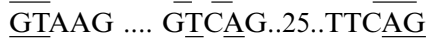 & GTTAT & 160 & 39.4 \\
\hline 11 & GAGGG & GTAAG .... CTTAC..31..TGCAG & CTACA & 107 & 41.1 \\
\hline 12 & GAAGA & 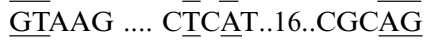 & GTTGG & 140 & 49.3 \\
\hline 13 & GTGTG & 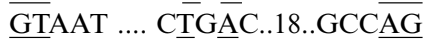 & GCTTA & 73 & 49.3 \\
\hline
\end{tabular}

${ }^{\mathrm{a}}$ Consensus sequences between introns are underlined.

${ }^{b}$ Numbers indicate number of nucleotides between adjacent sequences. 
environmental or physiological stimuli and are often associated with additional regions which possibly act as coupling elements determining signal response specificity (Menken et al., 1995).

Another notable feature derived from the sequence comparison is that the maize and rice Sbel genomic structures are quite similar. Both genes consist of 14 exons and 13 introns, the positions of which are conserved between the two species. The sizes of exons (exon 3-exon 13) constituting most of the mature proteins are identical except exon 5, in which the rice Sbel gene has one more codon compared with the maize gene. They share more than $86 \%$ and almost $90 \%$ similarity in nucleotide and amino acid sequences, respectively. However, exons not encoding the mature proteins (exon 1,2 and most of exon 14) do not display any significant sequence similarity and vary in size. The carboxylterminal 64 (67 in rice) amino acids encoded by exon 14 in the maize gene is the only region which is not conserved in the mature protein. Unlike the exons, homology was not found in any of the introns other than the splice junction sequences. The large intron (2212 bp) present in the rice Sbel gene is not found in the maize gene.

\subsection{Genomic Southern blot analysis}

To determine the number of Sbel genes in the maize genome, Southern blot analysis was performed. When blots were hybridized with the full-length maize Sbel cDNA (Fisher et al., 1995) under high-stringency conditions, at least three bands were detected in each lane (data not shown). Comparison of the hybridization patterns with the restriction map of the Sbel genomic clone (Fig. 1A) revealed that not all the bands in the Southern blot corresponded to the genomic map, suggesting more than one Sbel gene is present in the maize genome.

To confirm this, a $0.6-\mathrm{kb}$ genomic DNA probe which did not have any restriction enzyme sites used in the genomic blot was prepared from the genomic clone 25 1-1 by BamHI-HindIII digestion. The genomic probe will produce only one hybridizing band in every lane if there is a single copy of the Sbel gene in the maize genome. As shown in Fig. 5, however, the genomic probe (probe 2) containing the central region of the Sbel cDNA detected in each lane one or two additional bands apart from the bands predicted by the genomic map. This indicates that along with the isolated Sbe1 gene, another Sbel gene or a gene very closely related to Sbel exists in the maize genome. These will be referred to as Sbela (identified) and Sbelb (unidentified) to distinguish between them when appropriate. Interestingly, when a $1.7-\mathrm{kb}$ Bam HI-Pst I genomic fragment consisting of the Sbela promoter and transit peptide-coding region was used as a probe (probe 1),
A

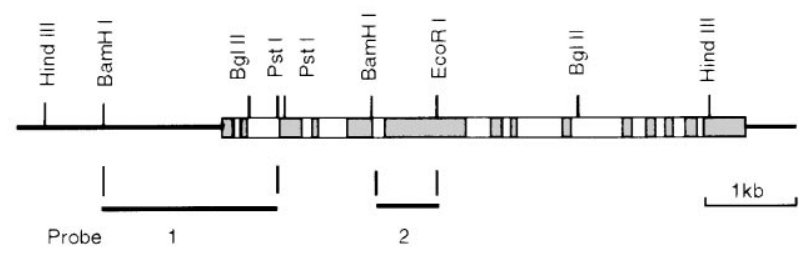

B

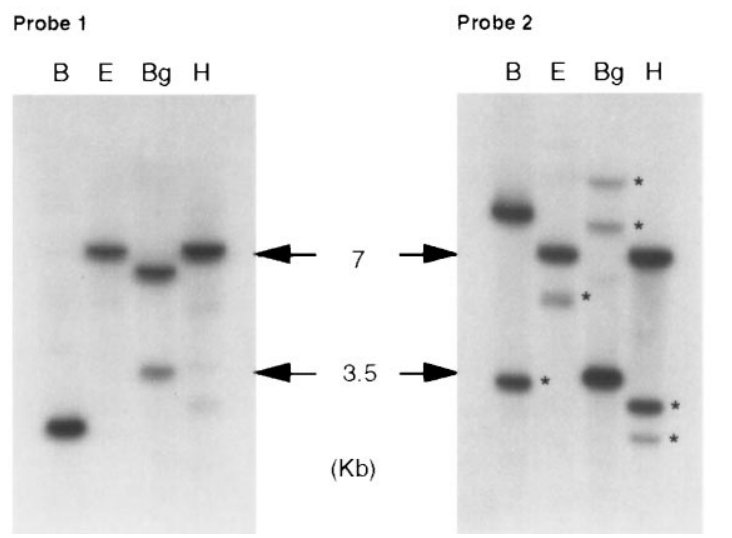

Fig. 5. Southern blot analysis of maize genomic DNA probed with partial Sbel genomic DNA fragments. (A) Schematic diagram of the Sbe1 genomic clone displaying the locations of restriction sites and the different probes used for genomic DNA analysis shown in (B). (B) Autoradiography of genomic DNA gel blot hybridization analysis. Each lane contains $10 \mu \mathrm{g}$ of maize genomic DNA digested with the indicated restriction enzymes: B, BamHI; E, EcoRI; Bg, BglII; H, HindIII. Blots 1 and 2 were probed at high stringency with probes 1 and 2, respectively. Bands which were not predicted from the restriction map of the Sbel genomic clone are indicated by asterisks on the right. Arrows indicate the position of the DNA size markers in kilobases.

only the bands predicted from the identified Sbela genomic DNA sequence were detected (Fig. 5). Taken together, these results suggest that although two Sbel genes are present in the maize genome, their 5 'flanking sequences and the $5^{\prime}$-end of the coding regions (at least at the DNA level) are quite divergent from each other.

\subsection{Genetic mapping of Sbe genes}

In order to map Shel loci onto the existing framework map (Gardiner et al., 1993), the full-length Sbel cDNA was used to probe a population of 54 immortalized $\mathrm{F}_{2}$ individuals from the cross Tx303 $\times \mathrm{CO} 159$ as part of the mapping efforts of the University of Missouri-Columbia Maize RFLP Laboratory. Two loci were identified on chromosome 6, bin 6.01 and chromosome 10, 10.04 . This supports the conclusion drawn above based on genomic Southern analysis that two Sbel genes exist in the maize genome. However, at present the data do not allow us to distinguish which gene is located on which chromosome. 


\subsection{Promoter activity}

A transient expression system was used to test whether the 5'-flanking sequence of the cloned Sbel gene ( $\lambda 5$ 1-1) is sufficient to support transcriptional activity. A chimeric gene containing a 2.2-kb 5' Sbel fragment $(-2191$ to +27$)$ fused to $\operatorname{UidA}$ reporter gene in pUC19 was first constructed and designated pKG101 (Fig. 6A). The chimeric plasmid was introduced into maize endosperm suspension cells via particle bombardment. Iodine staining and Northern blot analysis showed that the maize endosperm suspension cells actually produce starch and the genes involved in starch biosynthesis are expressed (data not shown). The bombarded cells were incubated for $24 \mathrm{~h}$ at $25^{\circ} \mathrm{C}$ in the dark and histochemical GUS assays were performed to visualize GUS expression. As shown in Fig. 6B, blue spots were observed, indicating that the 5'-flanking sequence has the ability to drive gene expression in the maize endosperm cells. Control cells bombarded with gold particles coated with promoterless UidA construct did not show any blue spots (data not shown). Further dissection of this promoter will be presented elsewhere.

A

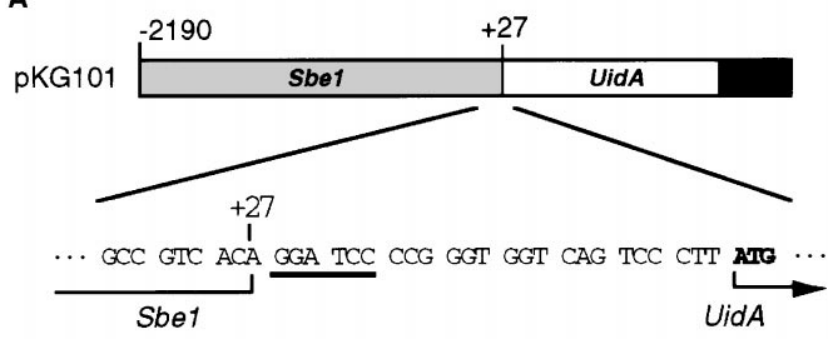

B

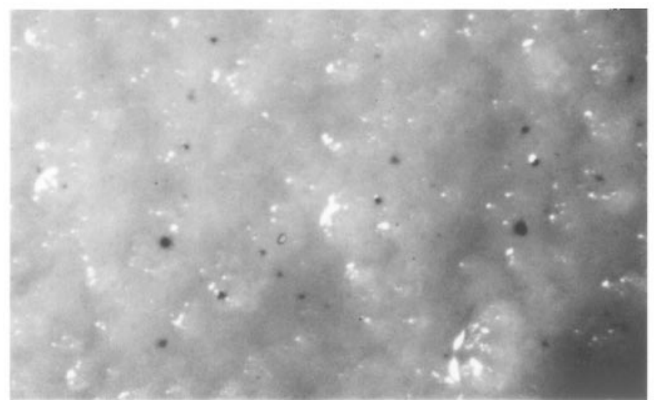

Fig. 6. Transient expression assay of the Sbe1 5'-flanking region in maize endosperm suspension cells. (A) Schematic diagram of the chimeric Sbel promoter-UidA construct. Numbers indicate distance relative to the Sbel transcription start site. Translation initiation starts at position +28 . The light grey (stippled) boxes indicates the Sbe1 promoter region. Open and solid black boxes represent UidA reporter gene and nopaline synthase 3 '-end sequences, respectively. The junction sequences between the Sbel gene and UidA are shown below the diagram. The BamHI sites used to join the two genes are underlined. The translation start site of UidA is indicated in bold. (B) Histochemical localization of GUS activity driven by the Sbe1 5'-flanking region in the maize endosperm suspension cells.

\section{Discussion}

Our previous work has shown that the maize Sbe genes exhibit both spatial and temporal modes of regulation. To begin to study the mechanisms regulating Sbe gene expression, a maize genomic library was screened with a ${ }^{32} \mathrm{P}$-labeled Sbel genomic PCR product corresponding to the region from 438 to 764 of the maize Sbel cDNA (Baba et al., 1991) and eight individual positive clones were isolated. Further analyses of the genomic clones indicated that they were all derived from a single locus, suggesting that only one Sbel gene might be present in the maize genome. However, genomic Southern analysis clearly indicated the presence of two Sbel genes. Genetic mapping also confirmed this conclusion, placing Sbel genes on both chromosomes 6 and 10 .

This discrepancy between the data can be explained by the results obtained from the genomic Southern blots probed with two different genomic DNA fragments (Fig. 5), which suggested the presence of two Sbel genes with divergent DNA sequences around the 5 -end of the coding region, as well as the $5^{\prime}$-flanking region. Since the PCR genomic fragment located near the 5 '-end of the Sbel cDNA was used as a probe for the library screening, it most likely failed to hybridize to the other Sbel gene. However, we cannot completely rule out the possibility that the second Sbel gene was not amplified during construction of the genomic library.

Based on hybridization analysis, it is noteworthy that the maize Sbel gene family consists of two genes which appear to have significantly different 5'-flanking sequences. This structural feature was also observed in another maize gene family ( $S h 1$ and $S u s l$ ) encoding sucrose synthase which is also involved in starch biosynthesis (Shaw et al., 1994). Like the Sbel genes, Shl and Sus 1 genes are markedly different from each other in their $5^{\prime}$-flanking regions in spite of striking similarities in their protein-coding regions. As might be expected, $S h 1$ and Susl genes are differentially regulated. The Shl gene is expressed mainly in endosperm, whereas the Susl gene is expressed in most maize tissues (Hannah and McCarty, 1988). In addition, the two genes responded to sugar levels in an opposite way (Koch et al., 1992). Low sugar concentrations increased the expression of the Shl gene, while high sugar levels favored accumulation of Susl mRNA. Therefore, it is possible that the two Sbel genes may display different expression patterns due to their divergent promoter regions. Cloning of the second Sbel gene will allow us to test this hypothesis.

The complete genomic structure of the isolated maize Sbela gene was established by DNA sequence analysis using one of the full-length genomic clones ( $25-1-1)$. Alignment of the genomic sequence with the Sbel cDNA revealed that the gene is composed of 14 exons and 13 introns. All the introns are flanked by the conserved 
splice junction sequences. Primer extension analysis and 5'-RACE determined the transcription start site and polyadeylation site and indicated that the transcribed region of the Sbel gene is $5690 \mathrm{bp}$ in length. A canonical TATA-box was found $25 \mathrm{bp}$ upstream of the transcription initiation site. In fact, we have shown that the Sbel 5 'flanking region $(-2190$ to +27$)$ containing the TATA-box has promoter activity in maize endosperm cells using a transient expression system.

Sequence comparison of the maize and rice Sbel genomic DNAs (Kawasaki et al., 1993b) revealed common structural features. First of all, both genes are composed of 14 exons and 13 introns. Second, the placement of all the introns are identical in both genes, yet their sizes and sequences are not conserved. This suggests that the introns are of ancient origin, although their sequences have evolved substantially since the divergence of maize and rice. Third, the exons in general exhibit striking similarity in size and sequence. The major differences are confined to the terminal exons such as exons 1, 2 and 14, which mostly do not encode mature protein. The carboxyl termini of the proteins are most divergent. The conservation patterns exhibited by the exons and introns of the two genes demonstrate that DNA sequences critical in gene function are evolutionarily conserved.

In this sense, two large highly conserved regions in the maize and rice Sbe1 5'-flanking sequences, which are located -2190 to -1890 and -1804 to -1611 in the maize Sbel gene, may have important roles in mechanisms controlling gene expression. In fact, our recent data showed that deletion of the regions resulted in a decrease in promoter activity in transient expression assays (K.-N. Kim, unpublished data). This suggests that they may act as distal positive regulatory elements in the Sbel gene.

Analysis of the amylose-extender (ae) maize mutant, which is now known to be the result of mutation in the Sbe2b gene (Fisher et al., 1993; Stinard et al., 1993), demonstrated the importance of SBEIIb activity in starch biosynthesis. The ae mutant endosperm was reported to produce almost $20 \%$ less total starch, which contains a higher percentage of amylose at the expense of amylopectin compared with the wild type (Shannon and Garwood, 1984). Unfortunately, however, no mutants deficient in the activity of SBEI have been reported so far, which makes it very difficult to appraise the significance of the SBEI contribution to starch biosynthesis. The presence of duplicate genes encoding SBEI may be one of the possible reasons explaining this situation. Otherwise, lack of such mutants could mean that loss of SBEI activity is lethal, or conversely it does not affect starch biosynthesis significantly enough to produce a detectable phenotype. Although the physiological importance of Sbel is unclear, the finding that the genomic organization of Sbel is evolutionarily con- served between maize and rice implies that at least in the monocots, Sbel should have an important function in starch biosynthesis, which is distinctive from other Sbe genes.

In conclusion, we demonstrated that two Shel genes are present in the maize genome. We also analyzed the genomic structure and the promoter region of the maize Sbela gene. These results should facilitate future studies of the regulatory mechanisms controlling its expression.

\section{Acknowledgement}

We would like to thank Dr. J. L. Anthony from DEKALB Genetics Corporation (Mystic, CT) for providing the maize endosperm suspension cells. We also appreciate Drs. Charles D. Boyer and Jack C. Shannon for their insight and support in this project. This work was supported by grants from Pioneer Hi-Bred International Inc. (Johnston, IA) to M.J.G., Charles D. Boyer and Jack C. Shannon and from the U.S. Department of Energy, Bioscience Program, to M.J.G., J.C.S., and Donald Thompson (DE-FG02-96ER20234) and by the Pennsylvania State Agricultural Experiment Station (No. CRIS 3303).

\section{References}

Baba, T., Kimura, K., Mizuno, K., Etoh, H., Ishida, Y., Shida, O., Arai, Y., 1991. Sequence conservation of the catalytic regions of amylolytic enzymes in maize branching enzyme-I. Biochem. Biophys. Res. Commun. 181, 87-94.

Boyer, C.D., Preiss, J., 1978a. Multiple forms of (1,4)- $\alpha$-D-glucan, $(1,4)-\alpha$-D-glucan-6-glucosyl transferase from developing Zea mays L. kernels. Carbohydr. Res. 61, 321-334.

Boyer, C.D., Preiss, J., 1978b. Multiple forms of starch branching enzyme of maize: evidence for independent genetic control. Biochem. Biophys. Res. Commun. 80, 169-175.

Brown, J.W.S., 1986. A catalogue of splice junction and putative branch point sequences from plant introns. Nucl. Acids Res. 14, 9549-9559.

Chisholm, D., 1988. A convenient moderate-scale procedure for obtaining DNA from bacteriophage Lambda. Biotechniques 7, 255-257.

Church, G.M., Gilbert, W., 1984. Genomic sequencing. Proc. Natl Acad. Sci. USA 81, 1991-1995.

Edwards, J., Green, J.H., Rees, T., 1988. Activity of branching enzyme as a cardinal feature of the ra locus in Pisum sativum. Phytochemistry 27, 1615-1620.

Fisher, D.K., Boyer, C.D., Hannah, L.C., 1993. Starch branching enzyme II from maize endosperm. Plant Physiol. 102, 1045-1046.

Fisher, D.K., Kim, K.-N., Gao, M., Boyer, C.D., Guiltinan, M.J., 1995. A cDNA encoding starch branching enzyme I from maize endosperm. Plant Physiol. 108, 1313-1314.

Fisher, D.K., Gao, M., Kim, K.-N., Boyer, C.D., Guiltinan, M.J., 1996. Allelic analysis of the maize amylose-extender locus suggests that independent genes encode starch-branching enzymes IIa and IIb. Plant Physiol. 110, 611-619.

Frohman, M.A., Dush, M.K., Martin, G.R., 1988. Rapid production of full-length cDNAs from rare transcripts: amplification using a 
single gene-specific oligonucleotide primer. Proc. Natl Acad. Sci. USA 85, 8998-9002.

Gao, M., Fisher, D.K., Kim, K.-N., Shannon, J.C., Guiltinan, M.J., 1996. Evolutionary conservation and expression patterns of maize starch branching enzyme I and IIb genes suggests isoform specialization. Plant Mol. Biol. 30, 1223-1232.

Gao, M., Fisher, D.K., Kim, K.-N., Shannon, J.C., Guiltinan, M.J., 1997. Independent genetic control of maize starch-branching enzymes IIa and IIb. Plant Physiol. 114, 69-78.

Gardiner, J.M., Coe, E.H., Melina-Hancock, S., Hoisington, D.A., Chao, S., 1993. Development of a core RFLP map in maize using an immortalized F2 population. Genetics 134, 917-930.

Guan, H.P., Preiss, J., 1993. Differentiation of the properties of the branching isozymes from maize (Zea mays). Plant Physiol. 102, $1269-1273$.

Hannah, L.C., McCarty, D.R., 1988. Mature pollen contains transcripts of the constitutive sucrose synthase (Css) gene. Maize Genet. Coop. NewsLett. 62, 59-60.

James, M.G., Robertson, D.S., Myers, A.M., 1995. Characterization of the maize gene sugary1, a determinant of starch composition in kernel. Plant Cell 7, 417-429.

Jefferson, R.A., 1987. Assaying chimeric genes in plants: the GUS gene fusion system. Plant Mol. Biol. Rep. 5, 387-405.

Junghans, H., Metzlaff, M., 1990. A simple and rapid method for the preparation of total plant DNA. Biotechniques 8, 176

Kainuma, K., 1988. Structure and chemistry of the starch granule. In: Preiss, J. (Ed.), The Biochemistry of Plants, Vol 14. Academic Press, San Diego, pp. 141-180.

Kawasaki, T., Hayashida, N., Baba, T., Shinozaki, K., Shimada, H., 1993a. The gene encoding a calcium-depedent protein kinase located near the sbe 1 gene encoding starch branching enzyme I is specifically expressed in developing rice seeds. Gene 129, 183-189.

Kawasaki, T., Mizuno, K., Baba, T., Shimada, H., 1993b. Molecular analysis of the gene encoding a rice starch branching enzyme. Mol. Gen. Genet. 237, 10-16.

Koch, K.E., Nolte, K.D., Duke, E.R., McCarty, D.R., Avigne, W.T., 1992. Sugar levels modulate differential expression of maize sucrose synthase genes. Plant Cell 4, 59-69.

Martin, C., Smith, A.M., 1995. Starch biosynthesis. Plant Cell 7, 971-985.

Menken, A.E., Schindler, U., Cashmore, A.R., 1995. The G-box: a ubiquitous regulatory DNA element in plants bound by the GBF family of bZip proteins. Trends Biol. Sci. 20, 506-510.

Mizuno, K., Kawasaki, T., Shimada, H., Satoh, H., Kobayashi, E., Okumura, S., Arai, Y., Baba, T., 1993. Alteration of the structural properties of starch components by the lack of an isoform of starch branching enzyme in rice seeds. J. Biol. Chem. 268, 19084-19091.

Murashige, T., Skoog, F., 1962. A revised medium for rapid growth and bioassays with tobacco tissue cultures. Physiol. Plant 15, 473-497.

Preiss, J., 1991. Biology and molecular biology of starch synthesis and its regulation. Oxford Surveys of Plant Molecular and Cell Biology 7, 59-114.

Rogers, S.O., Bendich, A.J., 1985. Extraction of DNA from milligram amounts of fresh, herbarium and mummified plant tissues. Plant Mol. Biol. 5, 69-76.

Salisbury, F.B., 1993. Gravitropism: changing ideas. Hortic. Rev. 15, 233-278.

Sambrook, J., Fritsch, E.F., Maniatis, T., 1989. Molecular Cloning: A Laboratory Manual, 2nd ed. Cold Spring Harbor Laboratory Press, Cold Spring Harbor, New York

Sanger, F., Nicklen, S., Coulson, A.R., 1977. DNA sequencing with chain-termination inhibitors. Proc. Natl Acad. Sci. USA 74, 5463-5467.

Shannon, J.C., Garwood, D.L., 1984. Genetics and physiology of starch development. In: Whistler, R.L., BeMiller, J.N., Paschall, E.F. (Eds.), Starch Chemistry and Technology. Academic Press, New York, pp. 25-86.

Shannon, J.C., Liu, J.W., 1977. A simplified medium for the growth of maize (Zea mays) endosperm tissue in suspension culture. Physiol. Plant 40, 285-291.

Shaw, J.R., Ferl, R.J., Baier, J., St.Clair, D., Carson, C., McCarty, D., Hannah, L.C., 1994. Structural feature of the maize sus1 gene and protein. Plant Physiol. 106, 1659-1665.

Smith, A.M., 1988. Major differences in isoforms of starch-branching enzyme between developing embryos of round- and wrinkled-seeded peas (Pisum sativum L.). Planta 175, 270-279.

Stinard, P.S., Robertson, D.S., Schnable, P.S., 1993. Genetic isolation, cloning, and analysis of Mutator- induced, dominant antimorph of the maize amylose-extender 1 locus. Plant Cell 5, 1555-1566.

Stitt, M., Huber, S., Kerr, P., 1987. Control of photosynthetic sucrose formation. In: Hatch, M., Boardman, N., (Eds.), The Biochemistry of Plants. Academic Press, New York, pp. 327-409

Takeda, Y., Shitaozono, T., Hizukuri, S., 1988. Molecular structure of corn starch. Starch/Staerke 40, 51-54.

Vries, S.D., Hoge, H., Bisseling, T., 1988. Isolation of total and polysomal RNA from plant tissues. Plant. Mol. Biol. Manual B6, 1-13.

White, P.J., 1994. Properties of corn starch. In: Hallauer, A.R. (Eds.), Specialty Corns. CRC Press, Ann Arbor, pp. 29-54.

Xu, R., Goldman, S., Coupe, S., Deikman, J., 1996. Ethylene control of E4 transcription during tomato fruit ripening involves two cooperative cis-elements. Plant Mol. Biol. 31, 1117-1127. 